\title{
Gastro Intestinal Stromal Tumor: State of the Art through Our Experience of 64 Cases and a Literature Review
}

\author{
Saad Slaiki*, Hicham El Bouhaddouti, Abdelmalek Ousadden, \\ Khalid Ait Taleb, El Bachir Benjelloun
}

Visceral Surgery Department, CHU Hassan II, Fes, Morocco

Email: *saad.slaiki@hotmail.com

How to cite this paper: Slaiki, S., El Bouhaddouti, H., Ousadden, A., Taleb, K.A. and Benjelloun, E.B. (2020) Gastro Intestinal Stromal Tumor: State of the Art through Our Experience of 64 Cases and a Literature Review. Journal of Cancer Therapy, 11, 265-275.

https://doi.org/10.4236/jct.2020.115022

Received: March 30, 2020

Accepted: May 5, 2020

Published: May 8, 2020

Copyright $\odot 2020$ by author(s) and Scientific Research Publishing Inc. This work is licensed under the Creative Commons Attribution International License (CC BY 4.0).

http://creativecommons.org/licenses/by/4.0/

\begin{abstract}
Gastrointestinal stromal tumors are rare. They are a subject of controversy. We have reported 64 cases of gastrointestinal stromal tumor diagnosed in the surgery department of CHU Hassan II of FES between January 2014 and December 2018. The study involved 64 patients ( 34 men and 30 women) with an average age of 56. The circumstances of findings were dominated by abdominal pain (48 cases), vomiting 16 case followed by transit disorder with 9 cases. The tumor locations were mainly the stomach $(\mathrm{n}=31)$, the small intestine $(n=28)$, the duodenum $(n=3)$, and the colon $(n=2)$. Ultrasound, endoscopy and CT were the main additional tests to detect tumor syndrome. 55 patients were treated by complete surgical excision. Tumor size ranged from $4 \mathrm{~cm}$ to $18 \mathrm{~cm}$. Histologically, the spindle cell type was predominant in $88.91 \%$ of cases; epithelioid type was present in $7.81 \%$ of cases, while the mixed type was found in 3.6\%. The analysis of the expression of CD 117 marker was present in $95.31 \%$, while immunostaining with this marker returned negative in 3 cases; i.e. $5 \%$ whose c-kit was positive. Imatinib was indicated in 44 patients (63.60\%), with 9 indications for metastatic tumor. As a neoadjuvant, imatinib was indicated in 5 patients, with remission in 28 patients (50.9\%), stabilization in 4 patients (7.2\%), 2 cases of tumor recurrence and 7 cases of death.
\end{abstract}

\section{Keywords}

Stromal Tumor, Imatinib, Surgery, GIST

\section{Introduction}

Gastro Intestinal Stromal Tumors (GIST) are rare. They can develop from all 
segments of the digestive tract, from the esophagus to the anus. These tumors have been the subject of multiple controversies; they have long been confused with other connective tumors. Nowadays due to the development of immunohistochemistry it is a precise and recent nosociological entity, grouping all digestive connective tumors derived from Cajal cells or one of their precursors and typically expressing the KIT + phenotype (95\% of case) and DOG- $1+(95 \%$ of cases) [1]. Their potential for malignancy is often difficult to assess. Stromal tumors are topical since the discovery of medical treatment of malignant forms with a tyrosine kinase inhibitor molecule (STI571 or GLIVEC). The surgical approach for GIST treatment, combining with molecular treatment makes it a multidisciplinary model of care [2]. We will try through the study of our observations of GIST collected in the department of visceral surgery in Hassan II Hospital in Fez, over a period of 5 years $(2014 / 2018)$ and a review of the literature to analyze the epidemiological aspects and to study the clinical and paraclinical characteristics as well as their therapeutic and evolutionary modalities to establish the precise diagnosis and to evaluate the therapeutic management of localized, advanced and inoperable tumors, and also to study the place of the surgical act as therapeutic basis of these tumors in the era of medical treatment that is GLIVEC.

\section{Methods}

- The type of study

This is a descriptive and comparative retrospective study of 64 patients.

- The framework of the study

It was carried out in the visceral surgery department of the Hassan II Fez University Hospital.

- The study period

It took place over a period of 05 years, from January 2014 to December 2018.

\section{- The study population}

* The inclusion criteria:

- Any patient presented in our department for diagnosis during the spread period from the year 2014 until the end of the year 2018 .

- Patients who have a GIST.

- Patients over the age of 16.

- Patients with designed histological evidence.

* Exclusion criteria:

- Any patient presenting outside the period already mentioned.

- Any patient with a histological type tumor other than GIST.

- The study of GIST in children, infants and newborns was excluded.

- Any patient who has not had histological evidence.

- Patients with incomplete records or insufficient data.

- Patients discharged against medical advice.

\section{- Data carriers}

The information was collected using an anonymous standardized operating 
sheet, using medical records, operative and anatomopathological reports. The analysis of the patients was made according to their age, sex, clinical presentation, location of the tumor, pathology, immunohistochemical characteristics, tumor extension and course of treatment.

\section{- Statistical analysis}

Data were captured, coded and analyzed in Excel.

\section{Result}

\section{- Patient characteristics}

The annual distribution of GIST cases is shown (Figure 1) with an average of 12.8 patients/year. The maximum number of cases was recorded in 2017 and 2018 with 22 and 16 patients respectively.

The age of our patients varied between 29 and 87 years with an average of 56.68 years. Regarding sex, we noted a clear male predominance with 34 men and 30 women, with a sex ratio of 1.13 .

\section{- Admission deadline}

The time between the onset of symptoms and the consultation was between 1 and 24 months. The majority of patients (78.12\%) consulted before the first year with a peak frequency of $48.43 \%$ between 01 month and 6 months (Figure 2).

\section{- Clinical and paraclinical presentation}

The clinical symptomatology was rich. Often each patient had several associated clinical signs (Figure 3).

The most commun sign was epigastralgia in 48 case i.e. $75 \%$ followed by vomiting in 16 cases, i.e. $21.8 \%$, deterioration of general condition and weight loss in 10 patients, i.e. $15.62 \%$, transit disorder in 9 cases, i.e. $14.06 \%$ and hemorrhagic syndrome: ( 6 cases had isolated hematemesis and 2 cases had melena). The gastric localization represented 31 cases among $64 \%$ or $48 \%$ of the cases, 28 patients had a small bowel localization or $44 \%, 3$ patients had a duodenal localization

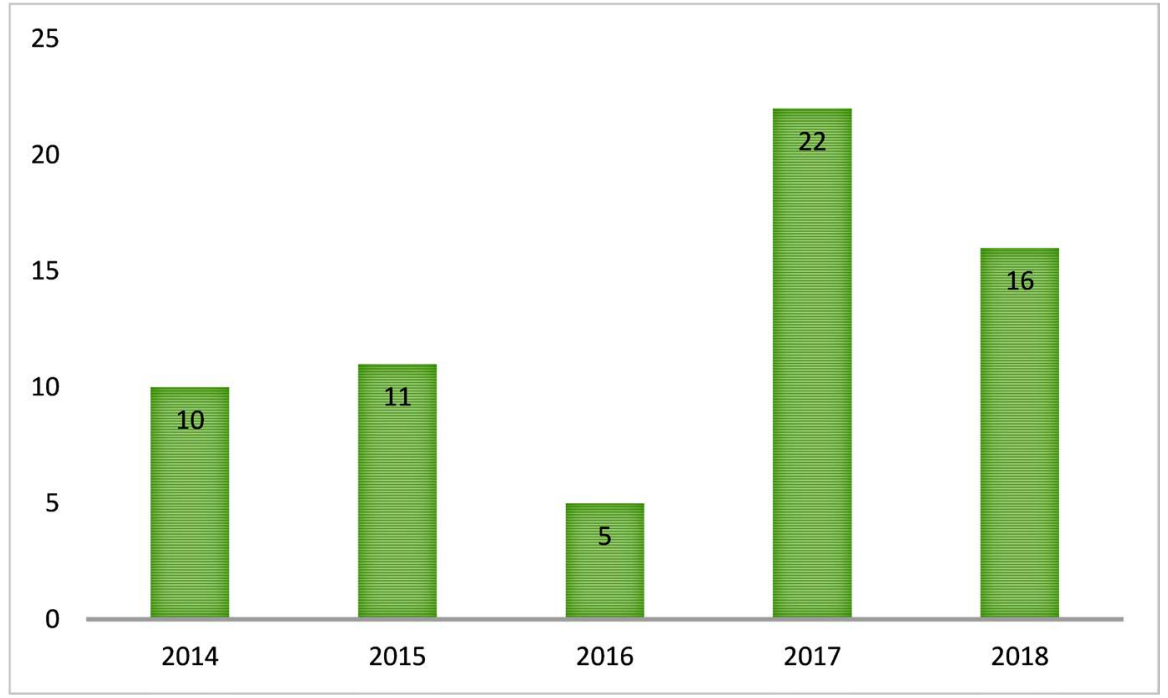

Figure 1. The evolution of the numbers of patients from the 2014 to 2018. 


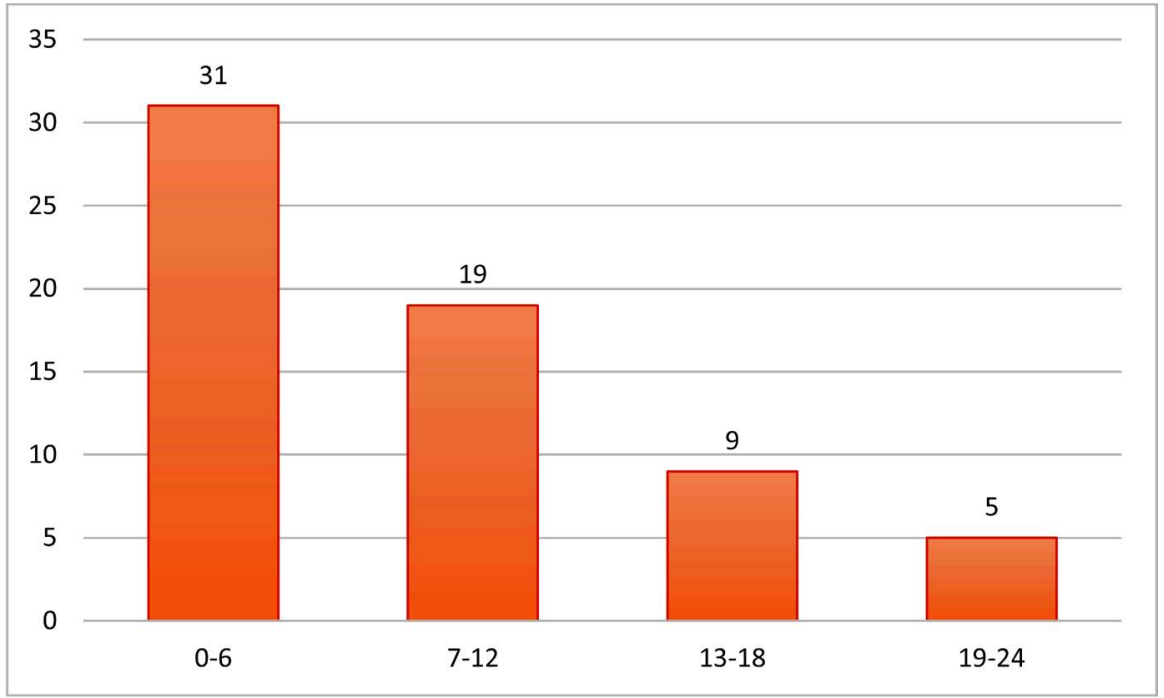

Figure 2. Patient diagnostic delays in our series.

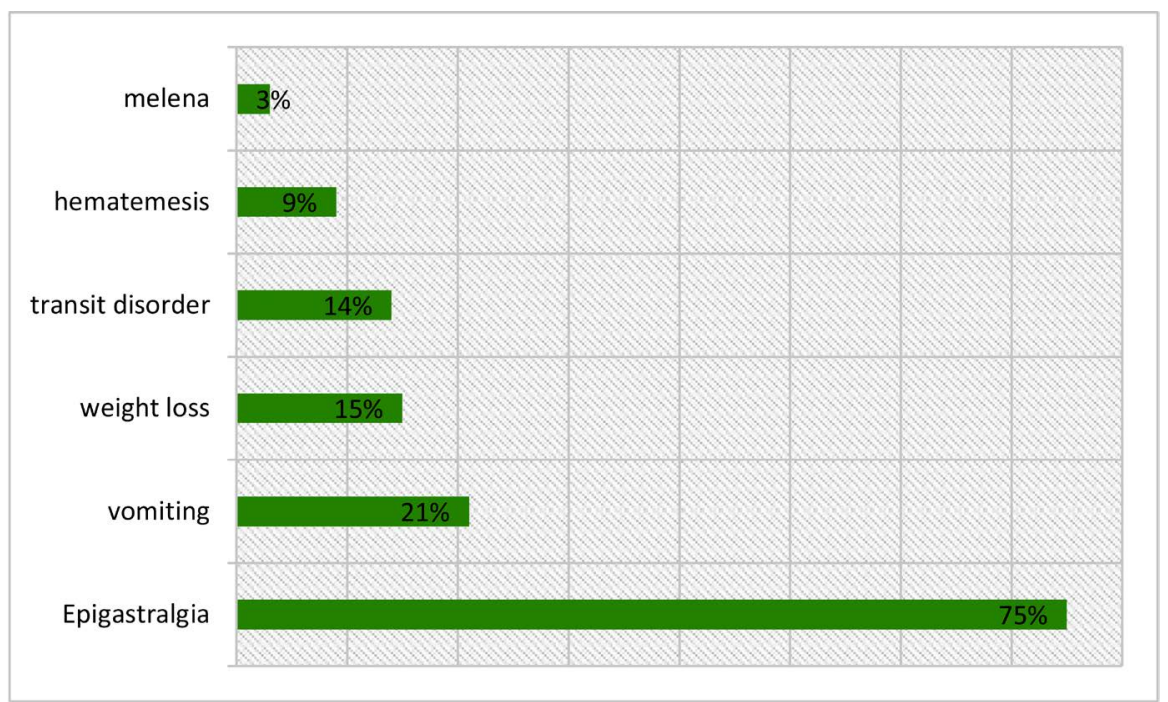

Figure 3. Distribution of patients according to clinical signs.

or $5 \%$ and 2 patients had a colonic localization or $3 \%$. Abdominal ultrasound was performed in 25 patients. It objectified a hypoechoic mass occupying the gastric wall or the intestinal wall. The CT scan was performed on all patients, allowed to visualize the mass with a sensitivity of $100 \%$. This mass was hypodense, heterogeneous depending on the gastric or intestinal wall, associated in 7 cases or $10.9 \%$ with hepatic metastases, in 2 cases or $3.12 \%$ with peritoneal metastases. Esogastroduodenal fibroscopy was performed in 34 of our patients, i.e. $53 \%$ of the cases, it was contributory in 26 cases and inconclusive in 8 cases. Biopsies were performed in all patients with local advanced or metastatic tumors. The tumors which were resected are the only ones which benefited from an evaluation of their sizes and their histopronostic grades, i.e. 55 cases. The tumor size varied between $3.9 \mathrm{~cm}$ and $18 \mathrm{~cm}$ with an average of $7.9 \mathrm{~cm}$. histologically, the spindle cell type was predominant, it was observed in $88.91 \%$ of cases, and the 
epithelioid type was present in $7.81 \%$ of cases, while the mixed type was found in $3.6 \%$.

In our series, the analysis of the expression of CD117 was made in all cases: $95.31 \%$ of the tumors showed a strong expression of CD117, while immunostaining with this marker returned negative in 3 cases, i.e. $5 \%$ whose c-kit was positive. In order to assess the degree of malignancy of the stromal tumors in our series, we mainly based on tumor size and location (Figure 4) (In all patients with resectable tumor) as well as on the mitotic index, i.e. 55 cases ( $\leq 5$ mitoses in 43 patients and $>5$ mitoses in 12 patients) (Figure 5).

\section{- Treatment and evolution}

In our series we had 55 (85.91\%) localized GIST and 9 (14.1\%) metastatic.

For localized tumor or 55 cases

All 55 patients were operated (Table 1), the surgical procedure was adapted to the tumor location and its locoregional extension. The resection was $\mathrm{R} 0$ in 50 patients or $90.09 \%, \mathrm{R} 1$ in 2 patients, i.e. $3.6 \%, \mathrm{R} 2$ in 3 patients or $5.4 \%$, postoperative course was uneventful in 43 patients (78.18\%), for the rest of patients

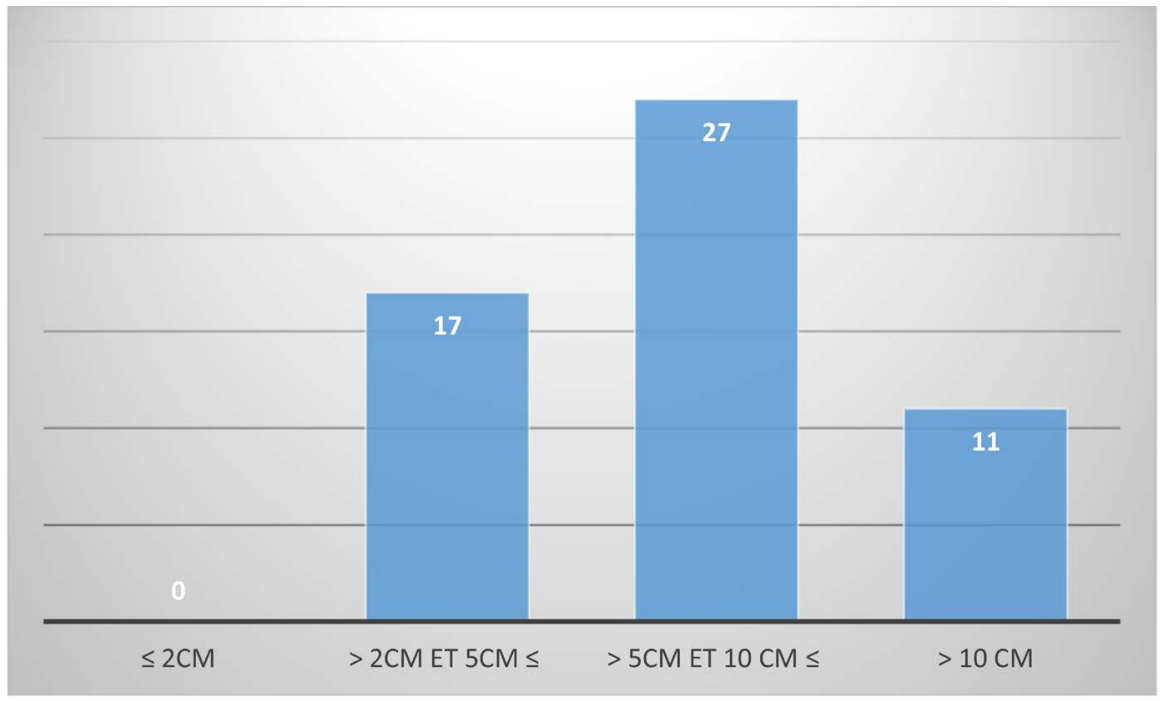

Figure 4. Distribution of cases according to tumor size.

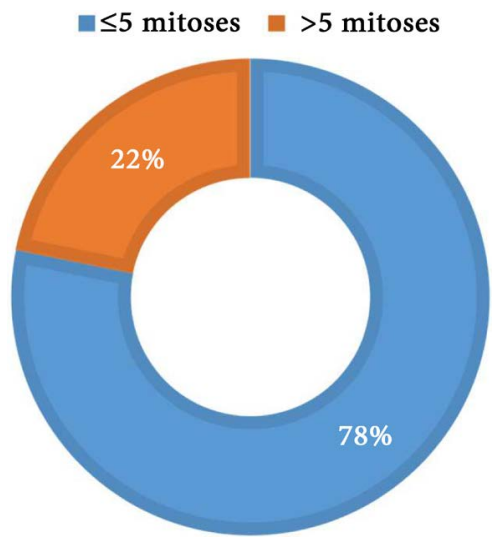

Figure 5. Distribution of cases according to mitotic index. 
Table 1. Type of surgery.

\begin{tabular}{cc}
\hline Surgical procedure & Number of patients \\
\hline Total gastrectomy & 3 patients $(5.40 \%)$ \\
Wedge resection & 25 patients $(39.06 \%)$ \\
Small bowel resection + anastomosis & 22 patients $(34.67 \%)$ \\
Colon resection & 2 patients $(3.6 \%)$ \\
Atypical duodenal resection & 2 patients $(3.6 \%)$ \\
Duodenal resection + anastomosis & 1 patient $(1.8 \%)$ \\
\hline
\end{tabular}

we had Infection of the wall: in 6 patients, i.e. $10.9 \%$, wall abscess in 2 patients, i.e. $3.6 \%$, urinary tract infection in 2 patients or $3.6 \%$, pneumopathies in a patient or $1.8 \%$, pulmonary embolism: in one patient (1.8\%). Imatinib was indicated in 40 of our patients, or $72.7 \%$ of cases, when combined with surgery, imatinib was indicated in 35 patients $(63.60 \%)$. The usual dose for adjuvant therapy was $400 \mathrm{mg} /$ day. An increase to $800 \mathrm{mg} /$ day in 3 patients was indicated. The Duration of adjuvant therapy varied between 3 and 72 months with an average of 28.65 months. As a neoadjuvant, imatinib was indicated in 5 patients, at a dose of $400 \mathrm{mg} /$ day for all patients. The duration of neoadjuvant treatment varied between 3 and 9 months with an average of 6 months. Tolerance to imatinib was generally good in our patients. 5 patients or $9.80 \%$ had side effects linked to imatinib. It was neutropenia in one patient, anemia in two patients, cytolysis in one patient, and nausea in one patient. Sunitinib was prescribed for 4 patients due to progression under imatinib. All operated patients benefited from regular clinical and radiological monitoring, with remission in 28 patients (50.9\%), stabilization in 4 patients (7.2\%), 2 patients, $3.6 \%$, presented a metastatic recurrence in the liver at 08 months and 15 months of evolution, one of whom died after a year of relapse, 4 patients presented a primary resistance to imatinib at a dose of 400 then $800 \mathrm{mg} / \mathrm{j}$ then on sunitinib and all died after a median follow-up of 7 months of progression, 17 were lost, i.e. $30.9 \%$.

For metastatic one

In our series we had 9 metastatic GIST: 2 with peritoneal metastasis and 7 with hepatic metastasis. This patient wasn't operated. We have started imatinib $400 \mathrm{mg} /$ day in all of them with stabilization of 3 cases, progression of 4 cases on imatinib $400 \mathrm{mg}$ and switch to sunitinib in 3 cases and 1 case to imatinib $800 \mathrm{mg}$ and death of 2 cases 6 months after treatment with a 12 month follow-up.

\section{Discussion}

gastro-intestinal stromal tumor are rare since they represent only $10 \%$ of soft tissue sarcomas and less than $1 \%$ of gastric tumors, $14 \%$ of intestinal tumors and $0.1 \%$ of colorectal tumors [3]. The recent recognition of this entity makes it difficult to assess its actual frequency. It is estimated, to date, that GIST affects approximately 15 people in 1 million, which represents approximately 800 to 900 
new cases per year in France [3], and approximately 10 - 15 per million per year in Western countries [4].

In Morocco, a study carried out in Marrakech objectified an incidence of $4.3 \%$ of gastric tumors [5]. Our study showed a frequency of $7.6 \%$ of gastric cancers therefore a little high compared to that of Marrakech. The incidence of these tumors by age is unimodal, with a peak frequency between 50 and 70 years and an average age of 58 years. The age of our patients varied between 29 and 87 years with an average of 56 years. GISTs are more frequent in men with a sex ratio of 1.5 to 2 , as is the case in our series with 34 men and 30 women, with a sex ratio $M / F$ of 1.13. No racial predominance is noted in the literature [3]. Gastric localization is the most frequent $(70 \%)$, followed by the small intestine $(20 \%$ $30 \%)$ and then come the colon (10\%), the rectum and even more rarely the esophagus and mesentery. Among the cases that we have reported Gastric localization represented 31 out of 64 cases or $48 \%$ of cases, 28 patients had a small bowel localization or $44 \%, 3$ patients had a duodenal localization i.e. $5 \%$ and 2 patients had a colonic localization or 3\% [6]. 30\% of GIST patients are incidental finding after performing an endoscopy, a sonography for another indication or during surgery for another disease [7]. 70\% are symptomatic of which $40 \%$ are revealed by a digestive hemorrhage and $10 \%$ due to intestinal perforation [8]. In our series pain was the main symptom (75\%) followed by vomiting $(21.8 \%)$, weight loss $(15.62 \%)$, transit disorder (14.06\%) only $12.5 \%$ had a hemorrhagic syndrome and no case presented in a table of peritonitis, this can be explained by the precocity of the delay of consultation, the majority of patients had consulted before the first year of their clinical symptoms. In $15 \%$ to $25 \%$ of cases, the disease is discovered at a metastatic stage [7]. In our series $10.9 \%$ had metastatic disease at the time of initial diagnosis. The diagnosis of GIST is based on endoscopic characteristics, echoendoscopic or radiological. However confirmation of the diagnosis of GIST is only histological [1]. The endoscopic appearance of GISTs is not very specific; generally we have a regular nodule, with a submucosal appearance because it is covered with normal mucosa. Endoscopic biopsies are most often negative, endoscopic resection to obtain a histological diagnosis is contraindicated because of the significant risk of perforation [6]. Echo-endoscopy is the best test to characterize oeso-gastro-duodenal or rectal submucosal lesions [3]. The echo-endoscopic appearance of GISTs is often typical: a hypoechoic, oval, homogeneous lesion with regular limits, developing from the fourth hypoechoic layer which corresponds to the muscularis [9]. The CT scan has a preponderant place. It allows to assess tumor extension. Generally, this examination is used to have more precision on the morphological characteristics of the tumor by determining its size, its homogeneity, the presence or not of areas of necrosis or areas of cystization [10]. MRI is an alternative to CT. it is more efficient than the scanner and avoids irradiation, by its multi-planar study capacities, allows a good study of the relationships of the tumor with the adjacent organs [11]. In our study tumors are characterized by the positivity of the CD117 marker in 
95.31\%. This immunohistochemical characteristic is similar to the data from the literature which reports good sensitivity of the CD117 marker with a positivity rates between $80 \%$ and $95 \%$ [12].

Surgery is the only potentially curative treatment for presumed resectable GISTs. The main objective of this process is to guarantee an adequate margin of resection without rupture of the tumor, nevertheless large margins have no advantage, there is no consensus on the distance of necessary safety, which can probably be $1 \mathrm{~cm}$ or less when the resection is R0. However, if the tumor adheres to an adjacent structure, en bloc resection taking away the adherent zone is the rule to prevent any tumor intrusion and to ensure complete resection. Lymph node dissection is not necessary due to the rarity of lymph node involvement. During the surgical procedure, an exploration of the liver and the parietal peritoneum is important in order to objectify possible metastases [3] [5]. On the other hand, imatinib can be indicated after multidisciplinary consultation when it is considered that it can modify the operative gesture by simplifying the surgery or by allowing a less mutilating resection. In our series, the surgery was R0 in 50 patients. Note that, five cases in this group (R0) were initially deemed unrespectable, and who responded favorably to neo-adjuvant treatment based on imatinib received for a period between 3 and 9 months. To evaluate the prognosis a histopronostic classification of GISTs was developed (Table 2) based on tumor size and the mitotic index so a tumor size greater than $2 \mathrm{~cm}$ is considered to be a factor of poor prognosis, the threshold of 5 mitoses makes it possible to differentiate tumors from low risk of malignancy [13]. However the threshold of these criteria varies according to the series and there is no international consensus [8]. There is no evidence to suggest that a specific surveillance protocol improves the prognosis. It is based on expert advice and must be adapted to the risk of recurrence, the terrain and whether or not prescription of an adjuvant treatment with imatinib (Figure 6). Exposure to ionizing radiation and its long-term risks must be taken into account, especially since the patient is young and the

Table 2. Estimated risk of recurrence in localized GIST resected [13].

\begin{tabular}{cccc}
\hline Malignancy risk & Tumor size $(\mathrm{cm})$ & Mitotic index (per 50 HPF) & Primary tumor site \\
\hline Very low & $\leq 2 \mathrm{~cm}$ & $\leq 5$ & Any \\
low & $>2-5 \mathrm{~cm}$ & $\leq 5$ & Any \\
Intermideate & $\leq 5 \mathrm{~cm}$ & $6-10$ & Gastric \\
& $>5-10 \mathrm{~cm}$ & $\leq 5$ & Gastric \\
High & Any & Any & Tumor rupture \\
& $>10 \mathrm{~cm}$ & Any & Any \\
& Any & $>10$ & Any \\
& $>5 \mathrm{~cm}$ & $>5$ & Any \\
& $\leq 5 \mathrm{~cm}$ & $>5$ & Non gastric \\
& $>5-10 \mathrm{~cm}$ & $\leq 5$ & Non gastric \\
\hline
\end{tabular}


risk of recurrence is low. An abdominal MRI is an alternative to CT [3]. For locally advanced, inoperable and or metastatic aggressive tumors, the prognosis has improved since the advent of imatinib $\left(\right.$ Glivec $\left.^{\circledR}\right)$. Its effectiveness is currently established; however the optimal methods of administration are not definitively determined. The daily dose recommended is $400 \mathrm{mg}$ per day, given until the patient's progression, intolerance or refusal (Figure 7) [3] [6].

In our study spread over 5 years has enabled us to determine the different

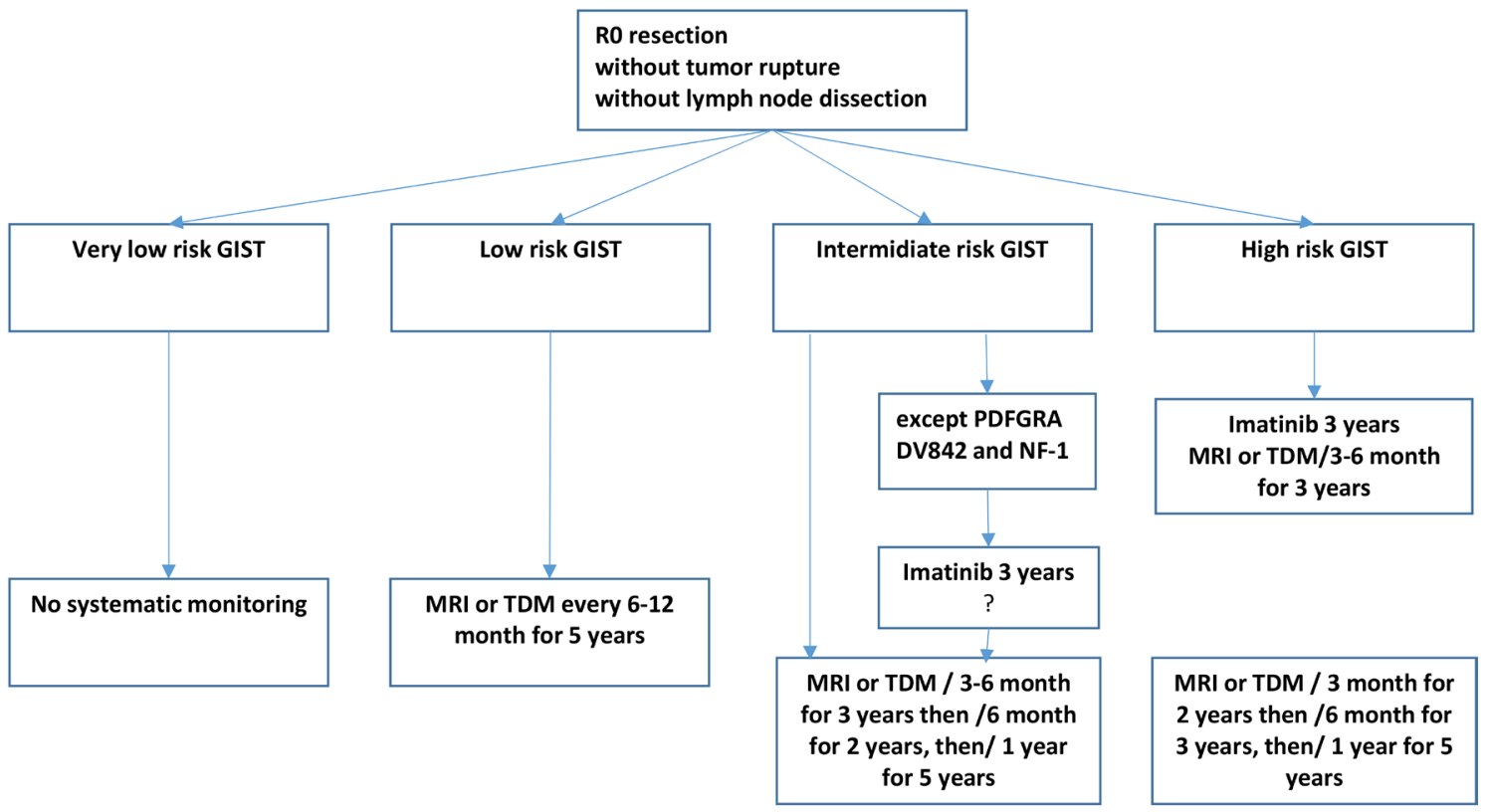

Figure 6. Localized GIST: management algorithm and surveillance [3].

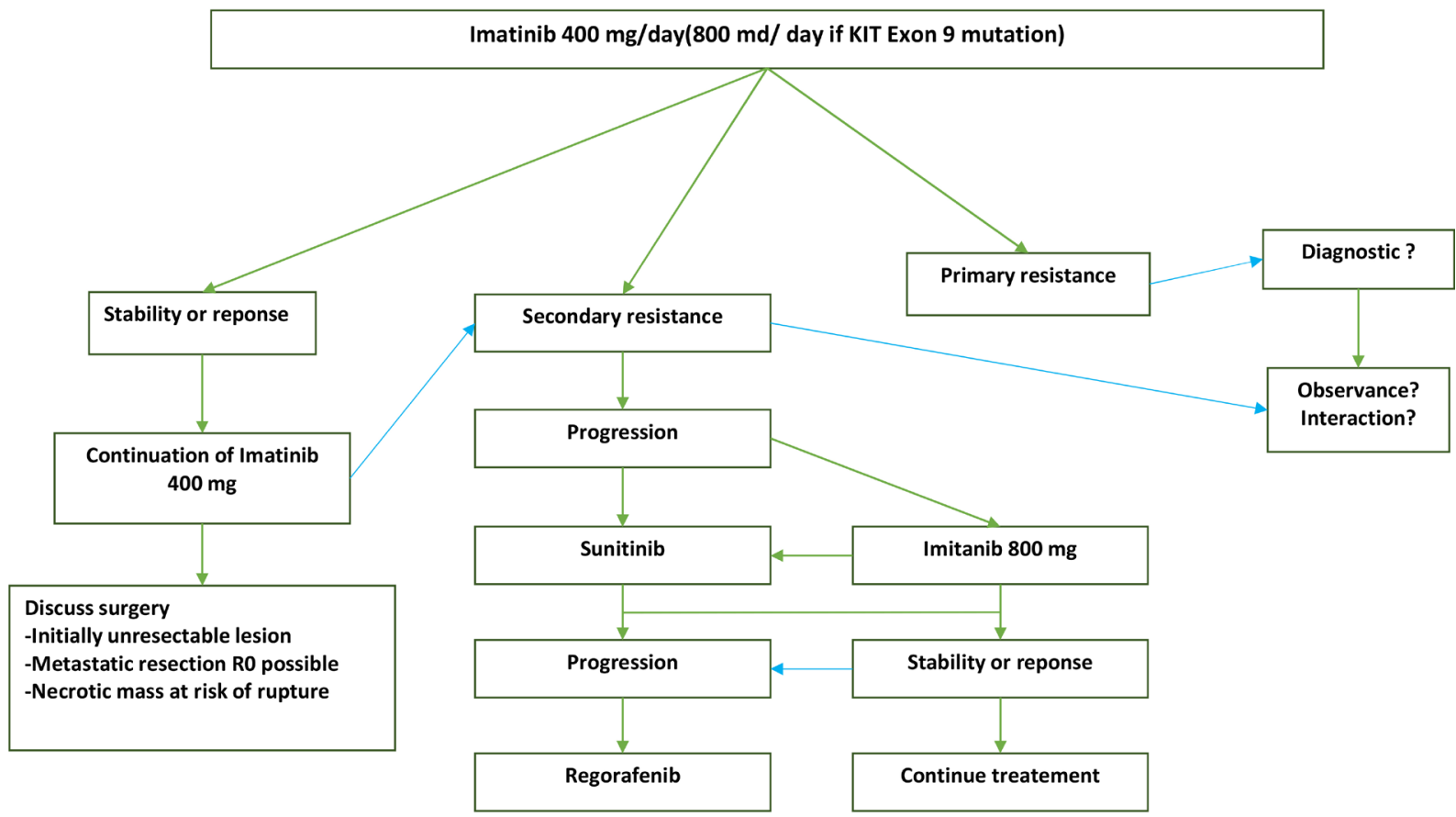

Figure 7. Management of metastatic or locally advanced GIST. 
epidemiologic, clinical, paraclinical and therapeutic aspects of this entity comparing to literature. However, the number of patients lost to follow-up compared to the total number and the duration of follow-up which was short over 12 months does not allow us to establish a prognosis. The continuation of our research work could also look into the prognostic aspect of this entity.

\section{Conclusion}

GISTs remain rare tumors in adults, mainly found in the stomach and small intestine, the diagnosis of which is histological. They occur secondarily to activating mutations of the KIT or PDGFRA receptors in $85 \%$ of the cases, and are diagnosed at a localized stage in approximately $85 \%$ of the cases. Surgery is the potentially curative treatment for localized GISTs. The histo-prognostic classifications taking into account the tumor location, the tumor diameter and the mitotic index make it possible to classify GISTs according to their risk of relapse and to set the stage for adjuvant treatment with imatinib. Imatinib, a tyrosine kinase inhibitor targeting at KIT and PDGFRA, completely changed the prognosis of these tumors. It is the only first-line treatment for metastatic or locally advanced GIST.

\section{Authors' Contributions}

All the authors testified to the care of the patient and the writing of the manuscript. The authors have read and approved the final version of the manuscript.

\section{Conflicts of Interest}

Authors do not declare any conflict of interest.

\section{References}

[1] Casali, P.G., Abecassis, N., Bauer, S., et al. (2018) Gastrointestinal Stromal Tumours: ESMO-EURACAN Clinical Practice Guidelines for Diagnosis, Treatment and Follow-Up. Annals of Oncology, 29, iv68-iv78.

[2] Demetri, G.D., Benjamin, R., Blanke, C.D., et al. (2004) NCCN Task Force Report: Optimal Management of Patients with Gastrointestinal Stromal Tumor (GIST) -Expansion and Update of NCCN Clinical Practice Guidelines. JNCCN, 2, S1-S26.

[3] Landi, B., Blay, J.-Y., Bonvalot, S., et al. (2019) Gastrointestinal Stromal Tumours (GISTs): French Intergroup Clinical Practice Guidelines for Diagnosis, Treatments and Follow-Up (SNFGE, FFCD, GERCOR, UNICANCER, SFCD, SFED, SFRO). Digestive and Liver Disease, 51, 1223-1231. https://doi.org/10.1016/j.dld.2019.07.006

[4] Joensuu, H., Eriksson, M., Sundby Hall, K., et al. (2012) One vs Three Years of Adjuvant Imatinib for Operable Gastrointestinal Stromal Tumor: A Randomized Trial. $J A M A, 307,1265-1272$. https://doi.org/10.1001/jama.2012.347

[5] Samlani-Sebbane, Z., Gharaba, S., Diffaa, A., et al. (2013) Les tumeurs stromales gastro-intestinales: Profil épidémiologique, clinique et thérapeutique au $\mathrm{CHU}$ de Marrakech. HEGEL_HEpato-Gastro Entérologie Libérale. https://doi.org/10.4267/2042/49207 
[6] Landi, B., Bouché, O., Guimbaud, R., et al. (2010) Tumeurs stromales gastro-intestinales (GIST) de taille limitée (inférieure à $5 \mathrm{~cm}$ ) : Revue de la littérature et propositions pour la prise en charge. https://www.em-consulte.com/en/article/244725 https://doi.org/10.1016/j.gcb.2009.06.019

[7] Rammohan, A., Sathyanesan, J., Rajendran, K., et al. (2013) A Gist of Gastrointestinal Stromal Tumors: A Review. World Journal of Gastrointestinal Oncology, 5, 102-112. https://doi.org/10.4251/wjgo.v5.i6.102

[8] Bonvalot, S. (2020) Traitement chirurgical des GIST à l'heure du Glivec ${ }^{\circledR}$. https://www.em-consulte.com/en/article/30080

[9] Landi, B. (2020) Tumeurs stromales gastro-intestinales (GIST) : Diagnostic et traitements (hors traitements endoscopiques).

https://www.fmcgastro.org/texte-postu/postu-2019-paris/gist-diagnostic-et-traiteme nts-hors-traitement-endoscopique/

[10] Michael King, D. (2005) The Radiology of Gastrointestinal Stromal Tumours (GIST). Cancer Imaging, 5, 150-156. https://doi.org/10.1102/1470-7330.2005.0109

[11] Blay, J.-Y., Landi, B., Bonvalot, S., et al. (2005) Recommendations for the Management of GIST Patients. Bull Cancer (Paris), 92, 907-918.

[12] Joensuu, H. (2006) Current Perspectives on the Epidemiology of Gastrointestinal Stromal Tumours. European Journal of Cancer, 4, 4-9. https://doi.org/10.1016/S1359-6349(06)80468-4

[13] Joensuu, H. (2008) Risk Stratification of Patients Diagnosed with Gastrointestinal Stromal Tumor. Human Pathology, 39, 1411-1419.

https://doi.org/10.1016/j.humpath.2008.06.025 